\title{
Wound contraction effects and antibacterial properties of Tualang honey on full-thickness burn wounds in rats in comparison to hydrofibre
}

\author{
Yan-Teng Khoo', Ahmad Sukari Halim", Kirnpal-Kaur B Singh², Noor-Ayunie Mohamad
}

\begin{abstract}
Background: Full-thickness burn wounds require excision and skin grafting. Multiple surgical procedures are inevitable in managing moderate to severe full-thickness burns. Wound bed preparations prior to surgery are necessary in order to prevent wound infection and promote wound healing. Honey can be used to treat burn wounds. However, not all the honey is the same. This study aims to evaluate the wound contraction and antibacterial properties of locally-produced Tualang honey on managing full-thickness burn wounds in vivo.
\end{abstract}

Methods: Thirty-six female Sprague Dawley rats were randomly divided into three groups. Under anaesthesia, three full-thickness burn wounds were created on the dorsum of the rats. The full-thickness burn wounds were inoculated with a specific organism $\left(10^{4}\right)$, namely Pseudomonas aeruginosa $(n=12)$, Klebsiella pneumoniae $(n=12)$, or Acinetobacter baumannii $(n=12)$. The three burn wounds were dressed with Tualang honey, hydrofibre and hydrofibre silver respectively. Swab samples were obtained every 3 days (day 3, 6, 9, 12, 15, 18 and 21) for quantitative and semi-quantitative microbiological analyses. Clinical assessments, including observations concerning the appearance and wound size, were measured at the same time.

Results: There was a rapid $32.26 \%$ reduction in wound size by day $6(p=0.008)$ in the Tualang honey-treated wounds, and $49.27 \%$ by day $15(p=0.005)$. The wounds remained smaller by day $18(p<0.032)$. Tualang honeytreated rats demonstrated a reduction in bacterial growth in Pseudomonas aeruginosa inoculated wounds $(p=0.005)$. However, hydrofibre silver and hydrofibre-treated wounds are superior to honey-treated wounds with Acinetobacter baumannii $(p=0.035)$. There was no statistical significant of antibacterial property in Klebsiella pneumonia inoculated wounds.

Conclusions: Tualang honey has better results with regards to its control of Pseudomonas aeruginosa and its wound contraction effects on full-thickness burn wound in vivo.

\section{Background}

Full-thickness burn wounds will not heal spontaneously, and inevitably need excision. Closure of the resultant wound is required in order to reduce the risks of invasive infection, which can result in systemic sepsis. In a majority of patients with full-thickness burns, early excision and immediate autografting is recommended [1]. In addition, it has been shown that full-thickness burn wounds are more commonly associated with infection than other tissue defects [2]. Infection of full-thickness

\footnotetext{
* Correspondence: ashalim@kb.usm.my

${ }^{1}$ Reconstructive Sciences Unit, School of Medical Sciences, Universiti Sains Malaysia, Malaysia

Full list of author information is available at the end of the article
}

burn wounds is frequently caused by bacterial organisms; the most common are Pseudomonas aeruginosa, Klebsiella pneumoniae and Acinetobacter baumannii. Given these problems, development of an alternative technique or dressing to reduce burn wound infection is of importance.

Honey can be a remedy for wound care. It is the oldest remedy for treating wounds, and dates back to the sixth century AD [3]. The ancient Egyptians used honey in a grease-honey-lint dressing to put on infected wounds. This traditional cure was displaced in the 1940 $\mathrm{s}$, before bacteria were discovered to be the cause of infection and with the discovery of antibiotics. It has recently been rediscovered by the medical profession, 
particularly where conventional modern therapeutic agents fail and with the trend of antibiotic-resistant wounds.

The current prevalence of antibiotic-resistant microbial species has led to a re-evaluation of the therapeutic use of ancient remedies such as honey. Honey has been reported to aid in wound healing, as it has special antibacterial and antibiotic properties [4]. More recently, honey has been reported to have an inhibitory effect on approximately 60 species of bacteria, including aerobes and anaerobes, gram-positive and gram-negative bacteria [5]. An antifungal action has also been observed for some yeast and species of Aspergillus and Penicillium, as well as all the common dermatophytes [5,6]. There are now many published reports describing the effectiveness of honey in rapidly clearing infections from wounds and protecting wounds from becoming infected [5,7-10]. Thus, it provides a moist healing environment without the risk of bacterial growth occurring, and with no adverse effects to slow the healing process. An infected wound will not heal unless bacteria are eliminated, as bacteria stimulate the inflammatory response, which can hinder the process of wound healing.

A honey dressing can undoubtedly be used on many types of wounds. Hence, previous study has produced an ideal wound dressing incorporating calcium alginate fibres and active Manuka honey [11]. It is believed that this alginate dressing offers antibacterial barrier protection and encourages wound healing. Many researchers have established the potency of Manuka honey to heal infected wounds and prevent bacterial infection [5,11]. However, not all honey is the same.

Tualang honey is a type of honey produced locally in Malaysia. The major components of Tualang honey are furfural derivatives such as 5-(hydroxymethyl)-furfural or HMF (25.4-185.6 mg/kg), furfural (46.9-58.5 mg/kg), 2-furylmethylketone $(0.2-0.9 \mathrm{mg} / \mathrm{kg}), 5$-methyl furfural $(2.2-3.6 \mathrm{mg} / \mathrm{kg})$ and fatty acids such as palmitic acid (341.0-531.4 mg/kg), ethyl linoleate $(2.0-46.7 \mathrm{mg} / \mathrm{kg}$ ) and ethyl oleate $(1.6-19.1 \mathrm{mg} / \mathrm{kg})$ [12]. A local study about the volatile components of Tualang honey has revealed 35 volatile compounds compared to other studies by Lušić and Odeh [13]. Odeh identified 30 compounds in Palestinian honey and Lušić identified 37 compounds from lime tree honey samples using solid phase microextraction (SPME) $[14,15]$. Tualang honey shares similar compounds, but it contains compounds that were not documented in literature before. They are terpenes and phynylethanal, which have significant results on microbiological activity. Terpene is a type of phytochemicals that contributes to the antimicrobial activity of honey [16]. Phenylethanal is an enzyme, reported to have some bactericidal effects.
Nuriza had performed an in vitro experiment on antibacterial activity of 5 types of Malaysian honey: Tualang, Hutan, Gelang, Pucuk Daun and Ee Feng Gu. Significant variation in composition of the honeys was noted. Tualang, Pucuk Daun and Ee Feng Gu honey had significant antibacterial activity against S.typhi, Staphy. aureus, S.Sonnie and E.coli in vitro [17].

Clostridium botulinum survives in honey, and therefore wound is at risk of botulism [18]. It is therefore recommended the honey to be sterilized by gamma irradiation at a dose of $25 \mathrm{kGy}$. Study has shown that radiation at 25 and $50 \mathrm{kGy}$ do not affect the quality of honey used [19].

The good wound healing properties of Malaysian honey is based on the previous studies of positive effect of honey on animal study, whereby the efficacy of using honey dressing was established [20,21]. To our knowledge, there is no published report on animal study testing specifically the efficacy of Tualang honey compared to hydrofibre silver on full thickness burn wound. This study performed a direct comparison with the most commonly used modern dressing in clinical practice. This study used Tualang honey which has not been studied using randomized method on full thickness burn wound; which represents a robust study design. Further research is needed to optimize the effective use of this agent in clinical practice. Nevertheless, its potency on wound healing and the effectiveness of its antibacterial properties can be studied in vivo before clinical use. This study was conducted to evaluate the wound healing and antibacterial properties of Tualang honey in fullthickness burn wounds in a Sprague Dawley rat model.

\section{Methods}

\section{Study design}

A randomized method was used for this animal study. This study was performed in the Laboratory of Animal Research Unit, Universiti Sains Malaysia. All the Sprague Dawley rats in this study were purchased from the animal centre of Hospital Universiti Sains Malaysia. The sample size was estimated using the two proportions formula with an $80 \%$ confidence interval. The sample size calculation was performed using the Power and Sample Size Calculation system (PS), with a type 1 error of 0.025 . A total of 36 rats were required for this study. The inclusion criteria included Sprague Dawley rats weighing between 250 and 350 grams.

\section{Experimental protocol}

All Sprague Dawley rats received humane care in compliance with the 'Principles of Laboratory Animal Care' formulated by the American Society for Medical Research and the 'Guide for the Care and Use of 
Laboratory Animals' prepared by the American Academy of Sciences and published by the National Institute of Health, United States of America. This study was approved by the university's animal ethics committee, USM Health campus with reference number of USM/ Animal Ethics Approval/2007/(34)(108).

The rats were housed individually in cages, and fed with free access to standard commercial rat food and water throughout the study. Thirty six rats was divided into 3 groups randomly i.e. 12 rats in each group (Group A, B \& C).

Tualang honey certified by Federal Agricultural Marketing Authority (FAMA), Malaysia, was used for wound dressing. This honey was collected in February and March of year 2009. This honey was sterilized by $\gamma$ irradiation at a dose of $25 \mathrm{kGy}$. The samples were stored in sealed, Teflon-coated plastic vials with silicone septa (Alltech, Milano, Italy) in a cool place before use. Hydrofibre and hydrofibre silver dressings used are plain Aquacel and Aquacel silver respectively. These dressings are produced by ConvaTec Inc, Skillman NJ 08558.

\section{Anaesthesia and surgical protocol}

On the day of wounding, the rats were placed in a ventral position and immobilized on their abdomen for the surgery. The dorsum of each rat was shaved. Immediately before the operation, the rats were anaesthetized with an intramuscular injection of $35.0 \mathrm{mg} / \mathrm{kg}$ Ketamine and $5.0 \mathrm{mg} / \mathrm{kg}$ Xylazine in the gluteal area. When fully anaesthetized, the shaved areas were cleaned with povidone iodine, alcohol and Hibiscrub ${ }^{\circ}$. The operation site was isolated with a sterile towel. Full-thickness burn wounds were created using sterile technique on the dorsum of the rats using hot metal heated with a burner at a temperature of $100^{\circ} \mathrm{C}$ for 30 seconds. A total of three full-thickness burn wounds were created on the dorsum of each rat. Each wound was $10 \mathrm{~mm}$ by $10 \mathrm{~mm}, 20 \mathrm{~mm}$ apart. The wound was inoculated with $10^{4}$ colony forming unit (CFU) of one of three common skin wound contaminants, namely Pseudomonas aeruginosa (Group $\mathrm{A}, \mathrm{n}=12$ ), Klebsiella pneumonia (Group B, $\mathrm{n}=12$ ) or Acinetobacter baumannii (Group C, $\mathrm{n}=12$ ). All the wounds in Group A ( $\mathrm{n}=12)$ were inoculated with Pseudomonas aeruginosa. All the wounds in Group B were inoculated with Klebsiella pneumonia. All the wounds in Group C were inoculated with Acinetobacter baumannii. All the rats (Group A, B \& C) received honey dressing on the first burn wound, hydrofibre on the second wound, and hydrofibre silver on the last wound. A thin layer of pure undiluted Tualang honey $(0.1 \mathrm{ml} /$ $\mathrm{cm}^{2}$ ) was applied topically to the first burn wound and filled up the wound. It was then covered with plain gauze. Hydrofibre and hydrofibre silver were applied to the other two wounds. All the dressing materials were covered with plain gauze separately. All the wounds were reinforced with crepe bandage. Thus, each group of rats received three wound dressings (Tualang honey, hydrofibre, and hydrofibre silver).

\section{Post-surgery care and follow up}

The animals were monitored immediately postoperatively for spontaneous breathing efforts and movement. After surgery, each animal was housed in an individual cage in a room and fed with standard rat diet and water, post-operative subcutaneous injection of morphine $0.02 \mathrm{mg} / \mathrm{kg}$ was given. All wounds were cleaned with normal saline and treatment reapplied every three days. All wounds were assessed clinically according to a scoring system (Table 1). The rats were then humanely euthanized with an intra-peritoneal injection of $5 \mathrm{mg}$ phenobarbitone sodium.

\section{Evaluation of wound size}

The wounds were subjected to evaluation every three days, i.e., on day 3, 6, 9, 12, 15, 18 and 21. Each wound was examined and photographs were taken after burn wound creation until healing was complete. Clinical assessments included observations concerning the appearance, and the wound size was measured using graph paper $\left(\mathrm{mm}^{2}\right)$. The wound size was measured from the periphery of the wound.

\section{Microbiological examinations}

Swabs were taken from the burn wound during each dressing change on day $3,6,9,12,15,18$ and 21 . The collected swabs were immediately sent to the laboratory for testing.

In the quantitative count study, $2 \mathrm{ml}$ of normal saline was added to each of the samples. The sample was vortexed thoroughly and a 10 -fold serial dilution was performed. Eight hundred microliters of each sample dilution was spread onto Tryptic Soy Agar (TSA). Two replicates were carried out for each dilution, and the agar plates were incubated at $37^{\circ} \mathrm{C}$ for 24 hours. The colonies were counted, and results were tabulated [22].

In the semi-quantitative analysis, swabs from wounds were spread onto blood agar plates according to the method described by Henry [23]. After 24 hours incubation at $37^{\circ} \mathrm{C}$, the blood agar plates were removed from the incubator and the growth of bacteria on each plate was scored as stated in Table 2 .

\section{Statistical analysis}

All the data were examined statistically. Data entry and analysis were done using Statistical Package for Social Sciences (SPSS) version 12.0. The values were expressed as Mean and Standard Deviation. Repeated measurements of Analysis of Variance (ANOVA) were used to compare mean differences and numerical data between 
Table 1 Dressing and wound evaluation system

\begin{tabular}{|c|c|c|c|c|}
\hline \multicolumn{5}{|l|}{ Dressing Evaluation } \\
\hline Flexibility & 0 & 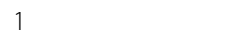 & 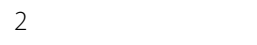 & 3 \\
\hline & (Not flexible) & (Minimal flexible) & (Moderate flexible) & (Flexible) \\
\hline \multirow[t]{2}{*}{ Adherence } & 0 & 1 & 2 & 3 \\
\hline & (Non-adherent) & (Minimal-adherent) & (Moderate-adherent) & (Good adherent) \\
\hline \multirow[t]{2}{*}{ Ease of removal } & 0 & 1 & 2 & 3 \\
\hline & (Very Difficult) & (Difficult) & (Easy) & (Very easy) \\
\hline \multirow[t]{2}{*}{ Fluid accumulation } & 0 & 1 & 2 & 3 \\
\hline & (Yes) & (Moderate) & (Minimal) & (No) \\
\hline \multicolumn{5}{|l|}{ Wound Evaluation } \\
\hline \multirow[t]{2}{*}{ Dryness of wound area } & 1 & 2 & 3 & \\
\hline & Wet & & Dry & \\
\hline \multirow[t]{2}{*}{ Exudation } & 1 & 2 & 3 & \\
\hline & Heavy exudates & Exudate & No exudates & \\
\hline \multirow[t]{2}{*}{ Wound Odour } & 1 & 2 & 3 & \\
\hline & Strong Odour & Mild Odour & Odourless & \\
\hline \multirow[t]{2}{*}{ Wound Contraction } & 1 & 2 & 3 & \\
\hline & Very Contracted & Contracted & Not Contracted & \\
\hline
\end{tabular}

and within groups, and the level of statistical significance was set at 0.05 .

Treatment groups were used as the independent grouping variable, and the wound contraction size $(\mathrm{mm})$ and the quantitative and semi-quantitative count of micro-organisms were used as the dependent variable.

The association between the dressing material and microbiological analysis was examined by non-parametric t-test. The final model of wound size used Kruskal-Wallis test. The $p$ value was significant, thus the model was fit. The main purpose of the model was to determine the interactions by using a two-way interactions test.

\section{Results}

\section{Clinical examinations}

Tualang honey dressing was more flexible, less adherent to the wound base, easier to peel off during dressing

Table 2 Semi-quantitative analysis scoring system for microbiological examinations

\begin{tabular}{llll}
\hline Score & \multicolumn{4}{l}{ Number of colonies in the streak area } \\
\cline { 2 - 4 } & $\mathbf{1}^{\text {st }}$ Quadrant & $\mathbf{2}^{\text {nd }}$ Quadrant & $\mathbf{3}^{\text {rd }}$ Quadrant \\
\hline $1+$ & $<10$ & $<5$ & \\
\hline $2+$ & $<10$ & $>5$ & $<5$ \\
\hline $3+$ & $>10$ & $>5$ & $>5$ \\
\hline $4+$ & $>10$ & & \\
\hline
\end{tabular}

changes and caused less fluid accumulation in the wound.

Comparison of the time course of post-burn wound sizes in the Tualang honey-treated wounds and hydrofibre silver-treated wounds showed that the mean wound size in the former group was statistically smaller. Wound size was found to be markedly reduced in the Tualang honey-treated wounds on day 3, 9 and 15 (Figure 1). The wounds showed a reduction in size of $12.86 \%$ by day 3 from the original $100 \mathrm{~mm}^{2}$ in the Tualang honey-treated wounds $(p=0.010)$. They further decreased in size of $33.94 \%$ by day 9 post-burn respectively (Figure 2). In the hydrofibre silver-treated wounds, the reduction from the original wound size was only $2.20 \%$ on day $3(p=0.010)$. The wounds were reduced by $13.74 \%$ by day $9(p=0.003)$. Over time, the reduction of wound size was shown in Figure 2. To determine the long term effect of Tualang honey on burn wounds, the wound healing process was observed up to 21 days. On day 21, honey-treated wounds in Pseudomonas aeruginosa inoculated group and Acinetobacter baumannii inoculated groups healed completely. The remaining wounds in Pseudomonas aeruginosa inoculated group and Acinetobacter baumannii inoculated groups and all the wounds in Klebsiella pneumonia inoculated wounds did not healed completely. 


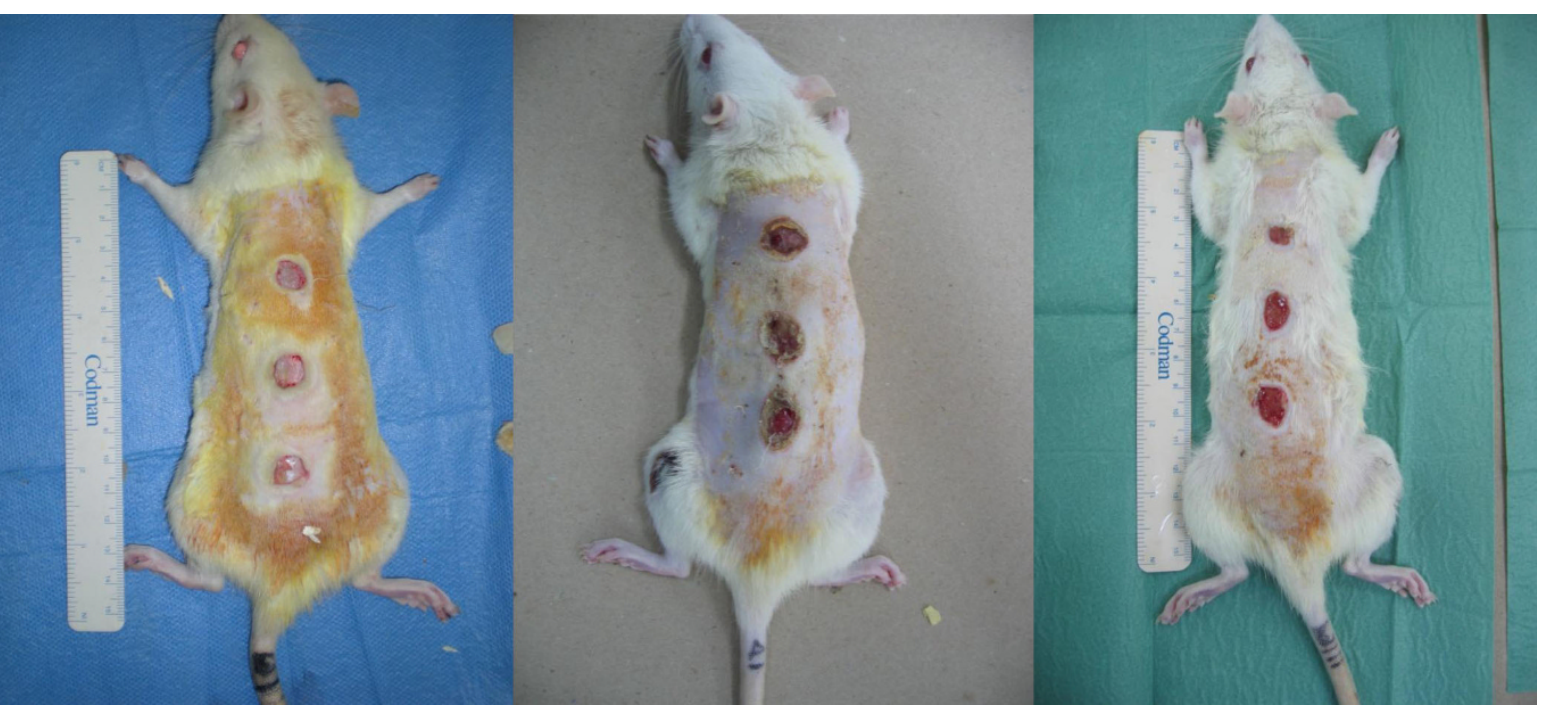

Figure 1 In Pseudomonas aeruginosa inoculated groups, post-burn wounds on day 3; day 9 and day 15.

\section{Microbiological examinations}

In the quantitative analysis, the Tualang honey-treated rats demonstrated a reduction in bacterial growth compared to hydrofibre silver and hydrofibre-treated wounds in Pseudomonas aeruginosa inoculated wounds ( $p=$ $0.005)$, with marked bacterial growth reductions on day $3,12,15,18$ and 21 of the experiment (Figure 3). However, hydrofibre silver and hydrofibre-treated wounds were superior to honey-treated wounds in Acinetobacter baumannii inoculated wounds $(p=0.035)$. There was no statistically significant difference between the three dressing materials used in Klebsiella pneumonia inoculated wounds.

The semi-quantitative examinations of the three organisms, Pseudomonas aeruginosa, Klebsiella pneumoniae and Acinetobacter baumannii, in the full-thickness burn wounds were performed in this study. In the Pseudomonas aeruginosa group, wounds treated with

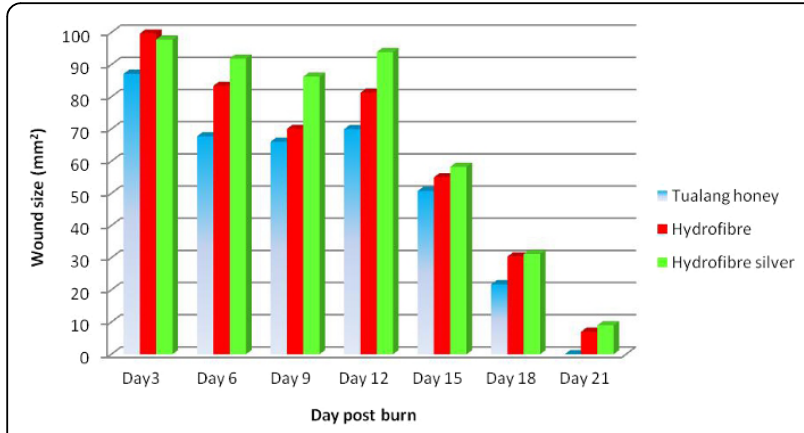

Figure 2 Comparison of wound size among three different dressing materials on burn wounds over time.
Tualang honey had decreased bacterial counts in the initial 6 days post-burn injury (day 3 and 6). By day 18 of the experiment, the bacterial growth was reduced to minimal in Tualang honey-treated and hydrofibre silvertreated wounds. There was no statistically significant difference in Klebsiella pneumoniae and Acinetobacter baumannii inoculated wounds.

\section{Discussions}

Many reports have been published about the usefulness of honey in wound management $[5,11,24,25]$. Honey is commonly utilized to dress wounds, for instance in burn wounds and other infected wounds [26]. Treatment of fresh wounds with honey was noted to produce increased wound contraction and an increase in granulation tissues [27]. Not all the honeys are the same; different types of honey have been used in many animal experiments to show acceleration in wound healing and its antibacterial properties $[17,19,28]$. The utility of Tualang honey for dressing burn wounds was demonstrated in this study.

The efficiency and efficacy of the topical application of honey on burns have been reported by previous study [21]. Molan found that the application of Manuka honey accelerated healing in acute wounds, chronic ulcers, burns, MRSA infections and infected wounds [29]. It shortens the healing period of the wounds. Hence, it is used in this study to test its properties on full-thickness burn wounds in an animal model. A report revealed that a honey dressing enhances wound contraction in fresh wounds, which is one of the key features of wound healing [27]. It is clear from this experiment that the in vivo wound contraction of burn 


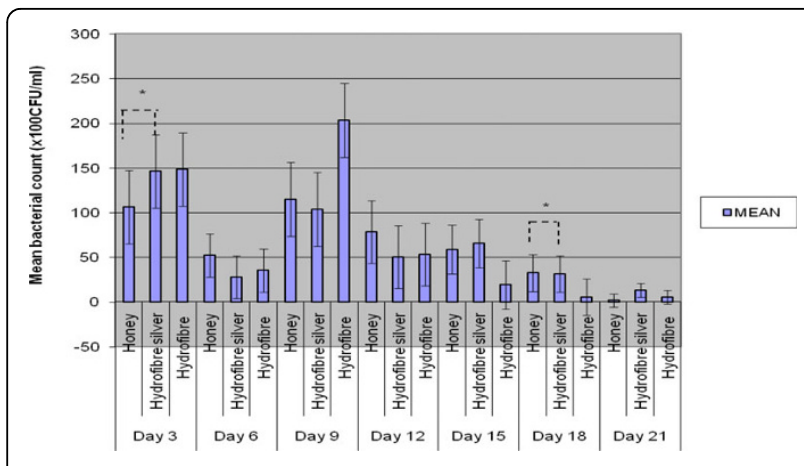

Figure 3 Comparison of colony count (CFU) among 3 wound dressings over time in Pseudomonas aeruginosa wounds. $n=36$, * $p<0.05$.

wounds dressed with Tualang honey was markedly greater than with the hydrofibre silver and hydrofibre dressing. It is especially true in the first 6 days postburn injury, when marked wound contraction was observed. The area of the wound was smaller, and epithelialization from the periphery of the wound edge was increased. The wound healing process was accelerated in the first 2 weeks in Tualang honey-treated wounds as compared to hydrofibre silver-treated wounds, throughout our experimental study. This shows that Tualang honey has the effect of promoting wound contraction in full-thickness burn wounds.

In this study, we noticed that the scabs formed by the honey-treated wounds were thinner compared to the other two treatment groups. It is believed that this condition could be attributed to the moist environment created by the honey due to its viscosity and high sugar content. Thus, thinner scabs form smaller barriers for the epithelialization to occur, and further accelerate the healing process. This observation indicates that the maximum wound contraction was in the Tualang honey group. This result was obtained by applying $0.1 \mathrm{ml} / \mathrm{cm}^{2}$ concentration of honey topically on the burn wounds. Our results suggest that Tualang honey applied topically to the burn wound accelerates the healing process. This will reduce the amount of autologous skin grafting necessary for full-thickness burn wound coverage, especially in minor burn.

After the creation of full-thickness burn wounds on Sprague Dawley rats, the Tualang honey was applied to the burned areas for 21 days, with treatment application every 3 days. Swab samples were collected at day 3, 6, 9, $12,15,18$ and 21 after the burn wound was created. The amount of the specific organism was analyzed using both quantitative and semi-quantitative methods. Molan demonstrated that Manuka honey has antibacterial properties against the Staphylococci and Pseudomonas aeruginosa [29]. There is report that honey is able to inhibit growth of both Gram-negative and Gram-positive organisms [30]. It is believed that the antimicrobial properties maybe due to hyper-tonicity and low $\mathrm{pH}$, as well as catalase in the honey, which inhibits the growth of microorganisms $[6,28]$. The effectiveness of honey as a dressing material can be attributed to its antimicrobial properties. The effectiveness of antibacterial properties of Malaysian Tualang honey was shown to be equivalent or better compared to Manuka honey in vitro [31]. Another study revealed that Tualang honey has comparable result for Gram-negative bacteria in-vitro [32]. The quantitative analysis of microbiological examination of this study has shown that Tualang honey-treated rats demonstrated a reduction in bacterial growth compared to hydrofibre silver and hydrofibre-treated wounds in Pseudomonas aeruginosa inoculated wounds $(p=0.005)$, with marked bacterial growth reductions. Semi-quantitative analysis of the Pseudomonas aeruginosa group showed that Tualang honey treated wounds had significant decreased bacterial counts in the initial 6 days post-burn injury. Both the quantitative and semi-quantitative analyses indicated that the Pseudomonas aeruginosa control by Tualang honey was superior compared with the other two dressing groups. In vitro study reported by Tan also showed that Tualang honey exhibit strong antibacterial activity against Pseudomonas aeruginosa [31]. This report showed the effectiveness of Tualang honey in controlling the proliferation of Pseudomonas aeruginosa. It can prevent burn wound infections in those Pseudomonas aeruginosa inoculated burn injury. The wound healing process will slow down if there is presence of underlying infection. The healing process will proceed orderly only when the infecting bacteria is cleared. This may further reduce the incidence of systemic sepsis and mortality. Previous studies reported that Manuka honey and honey pastures were effective in preventing the growth of Pseudomonas on the surface of wounds [33]. Local application of raw honey on infected wounds reduced the time for eradication of the bacterial infection due to Klebsiella $s p$ [34]. However, in this study, the overall poor antibacterial activity of Tualang honey against Klebsiella pneumoniae was unexpected in light of previous reports. The difference in results may be due to the different types of honey used in the studies. Previous studies used variable concentrations of honey to test the eradication of bacterial infections, and the present study used pure Tualang honey to determine its efficacy against Klebsiella pneumoniae. It is likely due to the variation in the composition of Tualang honey used in the present study. Part of the explanation may be that Klebsiella pneumoniae is a Gram-negative encapsulated organism, which reduces the penetration of honey.

Pseudomonas aeruginosa, Acinetobacter baumannii and Klebsiella pneumoniae are common pathogens that can cause nosocomial infections in a hospital setting. A study on the rate of infections in burns showed that 
in $65 \%$ of fatal burn cases, septicaemia was the cause of death, and Pseudomonas aeruginosa and Klebsiella sp. were the most common organisms. These organisms have resistance to many antibiotics and have become the predominant agents of wound sepsis in hospitals [35]. This study shows inhibition of Pseudomonas aeruginosa by Tualang honey with a topical application at a concentration of $0.1 \mathrm{ml} / \mathrm{cm}^{2}$. This shows the efficacy of antibacterial factors present in Tualang honey. Therefore, Tualang honey plays an important role in controlling the growth of bacteria, especially Pseudomonas aeruginosa, which may further avoid the burn wound infections that lead to systemic sepsis. The quantitative and semi-quantitative analyses indicated and supported that the Pseudomonas aeruginosa control by Tualang honey was superior compared with the other treatment groups. The relative benefit needs to be evaluated in clinical trials.

\section{Conclusions}

This experiment shows the positive effect of Tualang honey as a sound topical dressing for full-thickness burn wounds in an animal model. Tualang honey had better results with regard to its control of Pseudomonas aeruginosa infection and its wound contraction effects on burn wounds.

\section{Acknowledgements}

This work was supported by the Ministry of Higher Education Malaysia Universiti Sains Malaysia Research University (RU) Grant, (1001/PPSP/8120206) and Federal Agricultural Marketing Authority (FAMA), Kedah. Our thanks to the Laboratory Animal Research Unit for assistance provided and Dr. Kamarul Imran Musa who performed the statistical analysis.

\section{Author details}

${ }^{1}$ Reconstructive Sciences Unit, School of Medical Sciences, Universiti Sains Malaysia, Malaysia. ${ }^{2}$ Department of Medical Microbiology \& Parasitology, School of Medical Sciences, Universiti Sains Malaysia, Malaysia.

\section{Authors' contributions}

KYT carried out the animal experimental study, participated in the microbiological examination and drafted the manuscript. ASH designed the study and jointly drafted the manuscript. KKBS contributed to microbiological examination and analysis. MN and RAR contributed to the assistance for microbiological examinations. NAM contributed to laboratory work of histopathological examination. All authors read and approved the final manuscript.

\section{Competing interests}

The authors state that we have no competing interests. Each author has contributed original work to the manuscript and adhered to the ethical requirements as outlined by the journal.

Received: 16 April 2010 Accepted: 3 September 2010 Published: 3 September 2010

\section{References}

1. Wang JD: Early excision and one-stage grafting with full-thickness autologous skin in total deep burn of the face. Zhonghua Zheng Xing Shao Shang Wai Ke Za Zhi 1987, 3:103-105.
2. Smith DJ Jr, Thomson PD, Garner WL, Rodriguez JL: Burn wounds: infection and healing. American Journal of Surgery 1994, 167:46S-48S.

3. Golder W: Propolis. The bee glue as presented by the Graeco-Roman literature. Wurzbg Medizinhist Mitt 2004, 23:133-145.

4. Karayil S, Deshpande SD, Koppikar GV: Effect of honey on multidrug resistant organisms and its synergistic action with three common antibiotics. Journal Postgraduate Medicine 1998, 44:93-96.

5. Molan PC: Re-introducing honey in the management of wounds and ulcers - theory and practice. Ostomy Wound Manage 2002, 48:28-40.

6. Wilkinson JM, Cavanagh HM: Antibacterial activity of 13 honeys against Escherichia coli and Pseudomonas aeruginosa. J Med Food 2005, 8:100-103.

7. Cooper RA, Halas E, Molan PC: The efficacy of honey in inhibiting strains of Pseudomonas aeruginosa from infected burns. J Burn Care Rehabil 2002, 23:366-370.

8. Allen $\mathrm{KL}$, Molan PC, Reid GM: A survey of the antibacterial activity of some New Zealand honeys. J Pharmacol 1991, 43(12):817-822.

9. Willix DJ, Molan PC, Harfoot CG: A comparison of the sensitivity of wound-infecting species of bacteria to the antibacterial activity of manuka honey and other honey. J Appl Bacteriol 1992, 73(5):388-394.

10. Fruncillo RJ, DiGregorio GJ: The effect of thermal injury on drug metabolism in the rat. J Trauma 1983, 23(6):523-529.

11. Jull A, Walker N, Parag V, Molan PC, Rodgers A: Randomized clinical trial of honey-impregnated dressings for venous leg ulcers. Br J Surg 2008, 95:175-182.

12. Man Nin Che, Mohamed Mahaneem, Sulaiman Amrah Siti: The chemical compositions of Tualang honey. 2nd International Conference on the Medicinal Use of Honey, Kota Bharu, Malaysia. 13th - 16th 2010, Abstracts: P24.

13. Nurul Syazana NS, Gan SH, Halim AS: Volatile compositions of Malaysian Tualang (Koompasia Excelsa) honey. FP 13. 2nd International Conference on the Medicinal Use of Honey, Kota Bharu, Malaysia. 13th - 16th 2010

14. Odeh I, Abu-Lafi S, Dewik H, Al-Najjar I, Imam A, Valery M: A variety of volatile compounds as markers in Palestinian honey from Thymus capitatus, Thymelaea hirsuta, and Tolpis virgata. Food Chem 2007, 101(4):1393-1397.

15. Lušić D, Koprivnjak $O$, Curić D, Sabatini A: Volatile profile of Croatian lime tree (Tilia sp.), fir honeydew (Abies alba) and sage (Salvia officinalis) honey. Food Technol Biotechno 2007, 45:156-165.

16. Saravana KJ, Mandal M: Antiproliferative effects of honey and of its polyphenols: A review. Journal of Biomedicine and Biotechnology 2009, $1-13$.

17. Nuriza Tumin, Arsyayiah N, Halim A, Shahjahan M, Noor Izani N, Munavvar J, Sattar A, Hye Khan Abdul, Mohsin SSJ: Antibacterial activity of local Malaysian honey. Malaysian Journal of Pharmaceutical Sciences 2005, 2:1-10.

18. Snowdon JA, Cliver Do: Microorganisms in honey, review article. Int J Food Microbiol 1996, 31:1-26.

19. Yusof Norimah, Ainul Hafiza AH, Zohdi MRozaini, Bakar AZuki: Development of honey hydrogel dressing for enhanced wound healing. Radiation Physics and Chemistry 2007, 76:1767-1770.

20. Aljady AM, Kamaruddin MY, Jamal AM, Mohd Yassim MY: Biochemical study on the efficacy of Malaysian honey on inflicted wounds: an animal model. Medical Journal of Islamic Academy of Sciences 2000, 13(3):125-132.

21. Agata KD, Ewa SS, Robert DW, Artur S, Rafal S, Jerzy P, Jerzy S: Efficiency assessment of antimicrobial activity of honey-balm on experimental burn wounds. Bull Vet Inst Pulawy 2004, 48:109-112.

22. Qualitative and Quantitative Analysis in Microbiology. World of Microbiology and Immunology 2003, Encyclopedia.com.

23. Henry DI: Essential procedures for clinical microbiology. American Society for Microbiology Press Blackwell Science 1998.

24. Suguna I, Chandrakasan G, Ramamoorthy U, Joseph KT: Influence of honey on biochemical and biophysical parameters of wounds in rats. $J$ Clin Biochem Nutr 1993, 14:91-99.

25. Subrahmanyam M: A prospective randomized clinical and histological study of superficial burn wound healing with honey and silver sulfadiazine. Burns 1998, 24:157-161.

26. Dunford C, Cooper R, Molan PC, White R: The use of honey in wound management. Nurs Stand 2000, 15:63-68. 
27. Osuagwu FC, Oladejo OW, Imosemi IO: Enhanced wound contraction in fresh wounds dressed with honey in Wistar rats (Rattus Novergicus). West Afr J Med 2004, 23:114-118.

28. Bergman A, Yanai J, Weiss J, Bell D, David MP: Acceleration of wound healing by topical application of honey. An animal model. Am J Surg 1983, 145(3):374-376

29. Molan PC: Potential of honey in the treatment of wounds and burns. Am J Clin Dermatol 2001, 2:13-19.

30. Lusby PE, Coombes AL, Wilkinson JM: Bactericidal activity of different honeys against pathogenic bacteria. Arch Med Res 2005, 36:464-467.

31. Tan HZ, Rosliza AB, Gan SH, Halim AS, Hassan SA, Sulaiman SA, KirnpalKaur BS: The antibacterial properties of Malaysian tualang honey against wound and enteric microorganisms in comparison to manuka honey. BMC Complementary and Alternative Medicine 2009, 9(34):1-8.

32. Nasir NAM, Halim AS, Kirnpal-Kaur BS, Dorai AA, Haneef MNM: Antibacterial properties of tualang honey and its effect in burn wound management: a comparative study. BMC Complementary and Alternative Medicine 2010, 10(31):1-7.

33. Cooper R, Molan PC: The use of honey as an antiseptic in managing Pseudomonas infection. J Wound Care 1999, 8:161-164.

34. Al-Waili NS: Investigating the antimicrobial activity of natural honey and its effects on the pathogenic bacterial infections of surgical wounds and conjunctiva. J Med Food 2004, 7:210-222.

35. Sharma BR: Infection in patients with severe burns: causes and prevention thereof. Infect Dis Clin North Am 2007, 21:745-759.

Pre-publication history

The pre-publication history for this paper can be accessed here: http://www.biomedcentral.com/1472-6882/10/48/prepub

doi:10.1186/1472-6882-10-48

Cite this article as: Khoo et al.: Wound contraction effects and antibacterial properties of Tualang honey on full-thickness burn wounds in rats in comparison to hydrofibre. BMC Complementary and Alternative Medicine 2010 10:48.

\section{Submit your next manuscript to BioMed Central and take full advantage of:}

- Convenient online submission

- Thorough peer review

- No space constraints or color figure charges

- Immediate publication on acceptance

- Inclusion in PubMed, CAS, Scopus and Google Scholar

- Research which is freely available for redistribution

Submit your manuscript at www.biomedcentral.com/submit
Biomed Central 